\title{
Effects of Oxaliplatin and Oleic Acid Gc-Protein-Derived Macrophage- Activating Factor on Murine and Human Microglia
}

\author{
Jacopo J.V. Branca, ${ }^{1}$ Gabriele Morucci, ${ }^{1}$ Francesca Malentacchi, ${ }^{2}$ \\ Stefania Gelmini, ${ }^{2}$ Marco Ruggiero, ${ }^{2,3}$ and Stefania Pacini ${ }^{1} \star$ \\ ${ }^{1}$ Department of Experimental and Clinical Medicine, University of Firenze, Firenze, Italy \\ ${ }^{2}$ Department of Experimental and Clinical Biomedical Sciences, University of Firenze, Firenze, Italy \\ ${ }^{3}$ Immuno Biotech Ltd, St. Peter Port, Guernsey, Channel Islands
}

The biological properties and characteristics of microglia in rodents have been widely described, but little is known about these features in human microglia. Several murine microglial cell lines are used to investigate neurodegenerative and neuroinflammatory conditions; however, the extrapolation of the results to human conditions is frequently met with criticism because of the possibility of species-specific differences. This study compares the effects of oxaliplatin and of oleic acid Gc-protein-derived macrophage-activating factor (OA-GcMAF) on two microglial cell lines, murine BV-2 cells and human $\mathrm{C} 13 \mathrm{NJ}$ cells. Cell viability, cAMP levels, microglial activation, and vascular endothelial growth factor (VEGF) expression were evaluated. Our data demonstrate that oxaliplatin induced a significant decrease in cell viability in BV-2 and in C13NJ cells and that this effect was not reversed with OA-GcMAF treatment. The signal transduction pathway involving CAMP/VEGF was activated after treatment with oxaliplatin and/or OA-GcMAF in both cell lines. OAGcMAF induced a significant increase in microglia activation, as evidenced by the expression of the B7-2 protein, in $\mathrm{BV}-2$ as well as in $\mathrm{C} 13 \mathrm{NJ}$ cells that was not associated with a concomitant increase in cell number. Furthermore, the effects of oxaliplatin and OA-GcMAF on coculture morphology and apoptosis were evaluated. Oxaliplatininduced cell damage and apoptosis were nearly completely reversed by OA-GcMAF treatment in both BV-2/ SH-SY5Y and C13NJ/SH-SY5Y cocultures. Our data show that murine and human microglia share common signal transduction pathways and activation mechanisms, suggesting that the murine BV-2 cell line may represent an excellent model for studying human microglia. ๑ 2015 Wiley Periodicals, Inc.

Key words: oxaliplatin; OA-GcMAF; microglia

Resident microglia, known as the "macrophages of the central nervous system," may exert both pro- and anti-inflammatory effects (Romero-Sandoval et al., 2008). Microglia become activated by recognizing specific molecular patterns on pathogenic proteins and toxic debris during infections or nervous tissue injuries (Nguyen et al., 2002). Growing evidence ascribes some pathologic effects to glial cells, such as neuronal hyperexcitability and chronic inflammation induced by several toxicants and chemotherapeutic agents, including platinum-based drugs, taxanes, epothilones, and vinca alkaloids (Wolf et al., 2008). However, increasing numbers of studies provide evidence that microglial cells have many housekeeping functions that are necessary for healthy neuronal communication and neuroprotection (Marchand et al., 2005), contributing to a local immune environment that is beneficial for nearby healthy glial cells and neurons. Thus, it has been postulated that a delicate balance between pro- and anti-inflammatory events after damage is controlled by the finely tuned expression of "self-associated molecular pattern" and "altered self" proteins in microglia (Griffiths et al., 2007).

The intricacy of microglial biology is becoming more apparent, leading to a heightened research interest in this particular cell type. However, although properties of microglial cells have been well described in rodents, little is known about their human counterparts (Torres-Platas et al., 2014). Presently, many models of microglial cell lines, including primary cultures and immortalized cell lines are used to study neuroinflammatory and neurodegenerative phenomena. Quite obviously, the extrapolation of the results obtained in rodent microglial cells to their human counterparts may be debatable because, in

Contract grant sponsor: University of Firenze (to S.P., M.R.); Contract grant sponsor: Project PRIN 2009 (to S.P., M.R.).

*Correspondence to: Stefania Pacini, Department of Experimental and Clinical Medicine, University of Firenze, Largo Brambilla 3, 50134 Firenze, Italy. E-mail: stefania.pacini@unifi.it

Received 3 November 2014; Revised 11 February 2015; Accepted 26 February 2015

Published online 18 March 2015 in Wiley Online Library (wileyonlinelibrary.com). DOI: 10.1002/jnr.23588 
theory, the possibility of species-specific differences cannot be ruled out.

Therefore, this study was undertaken to assess these putative species-specific differences in the pathophysiological responses of rodent and human microglial cells. In particular, the focus of this study is to compare the effects of oxaliplatin, a well-known neurotoxic anticancer agent, and of oleic acid Gc-protein-derived macrophage-activating factor (OA-GcMAF), a protein inducing microglia activation (Morucci et al., 2014), on two microglial cell lines, murine BV-2 cells and human C13NJ cells, in terms of cell viability, cAMP levels, microglia activation, and vascular endothelial growth factor (VEGF) expression. Oxaliplatin was chosen for its well-studied mechanism of action and for its toxicity in neurons and glial cells. OAGcMAF was chosen because previous findings (Morucci et al., 2014) have demonstrated that it is possible to reverse the neuronal damage inflicted by oxaliplatin significantly and to preserve neuronal viability, possibly through a cAMP/VEGF neuroprotective signaling pathway. To investigate the role of oxaliplatin and OAGcMAF in microglial and neuronal signalling further and to study the interactions between rodent and human cell types, we developed cocultures of murine microglial cells/human neurons (BV-2/SH-SY5Y) and human microglial cells/human neurons (C13NJ/SH-SY5Y). We evaluated the morphology and apoptosis as consequences of the treatment with oxaliplatin and OA-GcMAF in these cocultures.

\section{MATERIALS AND METHODS}

\section{BV-2 Cell Line}

After successfully immortalizing murine macrophages from bone marrow via the v-raf/v-myc-carrying retrovirus (J2; Blasi et al., 1985), the Blasi research group adapted the same technique to obtain BV-2 microglia cells (Blasi et al., 1985, 1990). To develop this line, microglia were first purified by adhesion/agitation and then incubated overnight with control or J2 virus containing supernatants in cell-specific complete medium. After 3-4 weeks of incubation, proliferating cells were observed in the infected cultures, whereas noninfected cultures lost adherence and died (Blasi et al., 1990). The BV-2 cells were assessed for microglial cell markers; 90\% tested positive for nonspecific esterase activity, and all lacked peroxidase activity. Furthermore, BV-2 cells were positive for macrophage antigen (MAC) -1 and MAC-2 but were negative for MAC-3. Similarly to primary microglial cells, they were negative for glial fibrillary acidic protein (GFAP) and galactocerebroside antigens, which are markers for astrocytes and oligodendrocytes (Bignami et al., 1972), respectively. Analysis of cytokine secretion revealed that interleukin (IL) -1 increased in a dose-dependent manner when stimulated with lipopolysaccharide (LPS; Blasi et al., 1990). Additionally, given that these cells possess phagocytic capabilities, it was demonstrated that $A \beta(1-42)$ fibrils stimulated microglia-mediated phagocytosis in a time- and dose-dependent manner (Kopec and Caroll, 1998). Although they show similarities to primary microglia, the BV-2 cells do contain oncogenes that render them different from primary microglia in some ways. In particular, they show increased proliferation and adhesion and an increased variability of morphologies (Horvath et al., 2008). The validity of BV-2 cells as a sufficient substitute for primary microglia has been debated; this notwithstanding, only a few studies comparing different microglia lines have been published. One such study was conducted by Horvath et al. (2008), who compared primary rat microglia with the BV-2 line with regard to activation markers, motility, and releasable factors, such as nitric oxide (NO) and cytokines. It was observed that the primary microglia and BV-2 cells both expressed the ionized calcium binding adaptor molecule 1 (Iba1), a microglial activation marker. Furthermore, upon LPS stimulation, BV-2 cells secreted less but still substantial amounts of NO compared with primary microglia (Horvath et al., 2008). The hypothesis that BV-2-immortalized cells show features similar to those of primary microglia, even though they are not identical, was further examined by Henn et al. (2009). This group examined the BV-2 cells as an appropriate alternative to the primary cultures. They found that, in response to LPS, 90\% of genes induced in the BV-2 cells were also induced in primary microglia; however, the upregulation of genes in the BV-2 cells was far less pronounced than in primary microglia (Henn et al., 2009). In summary, for the BV-2 cell line, there appears to be a trade-off between the magnitude of the stimulatory response with the preparation time and technical feasibility between experiments.

\section{C13NJ Cell Line}

Primary cultures of human embryonic spinal cord and cortex were prepared from 8-10-week-old embryos as described by Peudenier et al. (1991). The cultures consisted of neurons, astrocytes, microglial cells, and fibroblasts. Adherent cells were selected and transfected by lipofection with PAS plasmid containing only the SV40 T antigen cDNA. Three weeks later, foci were independently isolated, expanded, and harvested for DNA analysis to demonstrate the presence of the integrated plasmid.

Monoclonal antibodies against CD68/ki-M7, CD68/ EBM11, CD11b/OKM1, 25F9 (a tissue macrophage marker), and anti-Fc receptors immunostained $64-98 \%$ of the transformed cells. These percentages are similar to those observed with primary microglial cells, with the exception of CD68/ EBM11, which was expressed in only $8 \%$ of the primary microglial cells. Two to nineteen percent of the cells, depending on the cell colony, phagocytized zymosan particles, with a maximum of 10 particles/cell. More than 95\% of cells displayed nonspecific esterase activity. The cells were not labeled with anti-CD68/KiM6, -CD14/leuM3, -CD11c/LeuM5, -MHCII/DR-2, or -CD4/Leu3a antibodies, which are low or absent on the membranes of primary microglial cells but are highly expressed on monocytes (control). The cells did not express class II major histocompatibility complex (MHCII) antigens spontaneously, but these antigens could be induced after stimulation of the transformed cells by human recombinant interferon- $\gamma$. Antigenic characteristics were retained after 35 passages. Finally, the absence of immunostaining with antiGFAP or antineurofilament antibodies in the cells indicated the 
absence of transformed astrocytes or neurons. Transformed cells as well as primary microglial cells produced IL-6.

Thus, the obtained transformed cells showed the macrophagic characteristics of adherence, phagocytosis, and intracytoplasmic nonspecific esterase activity and expressed several macrophagic antigens. The pattern of antigenic expression and cytokine secretion was identical to that of the primary microglial cells used for the transfection. Furthermore, the transformed cells could be activated with subsequent expression of MHCII antigens and IL-6 production (Peudenier et al., 1991). In conclusion, the transformed human microglial cells retained the properties of primary microglial cells, and they represent a homogeneous cell population that can be cultured indefinitely. The human microglial C13NJ cell line was kindly donated by Dr. Jean Mazella (Institut de Pharmacologie Moléculaire et Cellulaire).

\section{SH-SY5Y Cell Line}

The SH-SY5Y human neuron cell line was purchased from the Istituto Zooprofilattico dell'Emilia e della Romagna (Brescia, Italy). The SH-SY5Y cell line was chosen because of its dual role in oncology and neuropharmacology. This cell line, originally derived from a metastatic bone tumor biopsy (Kovalevich and Langford, 2013), represents a well-studied model for studying the effects of anticancer therapies targeted to neuroblastoma (Du et al., 2012; Sahu et al., 2013). However, this cell line also represents a valid model for studying the neurobiology of neurodegenerative diseases and neuropathies (Di Cesare Mannelli et al., 2013a), and it has been extensively used to study the neuroprotective effects of a variety of treatments (Thapa et al., 2013). The current study exploited these latter features of SH-SY5Y cells.

\section{Cell Culturing}

BV-2 cells were maintained in RPMI 1640 medium supplemented with 10\% fetal bovine serum (FBS) and $2 \mathrm{mM} \mathrm{L}-$ glutamine. C13NJ cells were cultured in Dulbecco's modified Eagle's medium (DMEM) supplemented with 10\% FBS and $2 \mathrm{mM}$ L-glutamine. SH-SY5Y neurons were maintained in DMEM/Ham's F12 supplemented with 10\% FBS and $2 \mathrm{mM}$ L-glutamine. Cells were maintained in a humidified atmosphere at $37^{\circ} \mathrm{C}$ in $95 \%$ air $/ 5 \% \mathrm{CO}_{2}$. The growth media were changed every 2-3 days. The BV-2/SH-SY5Y and C13NJ/ SH-SY5Y cocultures were maintained in DMEM supplemented with 10\% FBS and $2 \mathrm{mM} \mathrm{L}$-glutamine. Cell media, FBS, and L-glutamine were purchased from EuroClone (Milan, Italy). Before each treatment (see Results for further description), the cell lines were serum starved for $24 \mathrm{hr}$ in the appropriate media without FBS (starvation medium). After $24 \mathrm{hr}$ of starvation, fresh starvation medium containing the stimuli described below was added for 24 or $48 \mathrm{hr}$.

\section{Stimuli}

Oxaliplatin (Sigma Aldrich, Milan, Italy), was dissolved in phosphate-buffered saline (PBS) and used at concentrations of 1 $\mu \mathrm{M}, 10 \mu \mathrm{M}, 100 \mu \mathrm{M}, 500 \mu \mathrm{M}$, and $1 \mathrm{mM}$. OA-GcMAF was kindly donated by Immuno Biotech (Guernsey, Channel
Islands). OA-GcMAF was prepared as described elsewhere (Knyazeva et al., 2008; Morucci et al., 2014). OA-GcMAF showed a typical dose-dependent effect on all the different cell lines used to test its efficacy. The $800 \mathrm{pM}$ concentration was chosen because it was the highest concentration that induced a positive cellular response just before reaching the plateau doses and because higher concentrations did not show any additional effect.

\section{Cell Viability Assay}

Cell viability was evaluated by the reduction of a tetrazolium salt, 2-(2-methoxy-4-nitrophenyl) -3-(4-nitrophenyl) -5(2,4-disulf-ophenyl) $-2 \mathrm{H}$ tetrazolium monosodium salt (WST8 ), as an indicator of cellular dehydrogenase activity. BV-2 and C13NJ microglial cells were seeded into 96-well plates at densities of 3,000 cells/well and 10,000 cells/well, respectively, in their appropriate complete growth media. Cells were starved for $24 \mathrm{hr}$ and then treated with different concentrations of oxaliplatin and/or OA-GcMAF. Because of the stronger resistance of $\mathrm{C} 13 \mathrm{NJ}$ cells to oxaliplatin-induced damage, the oxaliplatin concentrations used on this cell line were higher than those used for the BV-2 cells. Cells were treated with oxaliplatin alone for $24 \mathrm{hr}$ and with OA-GcMAF alone for $48 \mathrm{hr}$. When the neuroprotective effects of OA-GcMAF were investigated, OA-GcMAF was added to the starvation cell medium $24 \mathrm{hr}$ before oxaliplatin, which was then added for an additional 24 $\mathrm{hr}$. In this case, OA-GcMAF remained in the culture media for a total of $48 \mathrm{hr}$. At the end of each treatment, the media were replaced with $100 \mu \mathrm{l}$ fresh starvation media, and $10 \mu \mathrm{l}$ chromogenic substrate (WST-8) was added to each well. After incubating the wells for $3 \mathrm{hr}$ at $37^{\circ} \mathrm{C}$, the absorbance was directly measured at $450 \mathrm{~nm}$ (optical density) with a Multiscan FC photometer (Thermo Scientific, Milan, Italy). Each experimental point was performed in quintuplicate, and each experiment was performed three times.

\section{Cell Density and Morphology Evaluation}

BV-2 and C13NJ microglial cells were seeded at 30,000 cells/coverslip in their appropriate complete growth media. Cells were starved for $24 \mathrm{hr}$ and then treated with different concentrations of oxaliplatin and/or OA-GcMAF for 24 or $48 \mathrm{hr}$.

At the end of the treatments, the media were removed and the cells were washed twice with cold PBS. One milliliter of $0.5 \%$ paraformaldehyde was added to each coverslip for 10 min. After two washes with cold PBS, the coverslips were dried for $1 \mathrm{hr}$ at room temperature and then stained with $\mathrm{H} \& \mathrm{E}$ dye following a standard procedure. Coverslips were observed via light microscopy, and images were taken at different magnifications with a digital camera (Nikon Coolpix 990; Nikon Instrument, Milan, Italy). Quantification of cell density was performed by measuring the cell surface $/ \mathrm{mm}^{2}$ in Image (NIH, http://imagej.nih.gov/ij, 1.47t).

\section{cAMP Assay}

The BV-2 and C13NJ microglial cell lines (both at the density of $4 \times 10^{6}$ cell/well) were plated in Petri dishes in their 
appropriate complete growth media. After $24 \mathrm{hr}$ of starvation, cells were treated for $25 \mathrm{~min}$ with oxaliplatin and/or OAGcMAF. Then, the medium was removed and $1.5 \mathrm{ml}$ of $\mathrm{HCl}$ (0.1 M) was added for $20 \mathrm{~min}$ to allow cell lysis and phosphodiesterase degradation. Cells were collected by scraping, and the supernatant was obtained by centrifugation at room temperature for $10 \mathrm{~min}$ at $1,800 \mathrm{rpm}$. A cAMP-competitive ELISA assay (Thermo Scientific) was performed on the supernatants to assess cAMP formation in response to different stimuli.

\section{Western Blotting}

The BV-2 and C13NJ microglial cell lines (both at the density of $4 \times 10^{6}$ cell/well) were plated in Petri dishes in their appropriate complete growth media. After $24 \mathrm{hr}$ of starvation, cells were treated for 24 or $48 \mathrm{hr}$ with oxaliplatin and/or OAGcMAF. Then, the medium was removed, and two washes with PBS were performed before cells were scraped from the surface of the dishes. The cell suspensions were centrifuged at 1,000 rpm for $10 \mathrm{~min}$ at room temperature. The pellets were treated with lysis buffer containing protease inhibitors (Sigma Aldrich) for 30 $\min$ at $4^{\circ} \mathrm{C}$, vortexing every $5 \mathrm{~min}$. The homogenates were centrifuged at $4^{\circ} \mathrm{C}$ for $10 \mathrm{~min}$ at $12,000 \mathrm{rpm}$, and the supernatants were used to evaluate protein concentrations by the Bradford method. Equal amounts of protein $(30 \mu \mathrm{g})$ were analyzed on a $12 \%$ polyacrylamide gel and then transferred onto nitrocellulose membrane (Porablot NPC; Macherey-Nagel, Carlo Erba Reagents, Milano, Italy). After having been blocked for $1 \mathrm{hr}$ at room temperature with $5 \%$ bovine serum albumin in Trisbuffered saline (TBS) containing $0.1 \%$ Tween 20, the blot was incubated overnight at $4^{\circ} \mathrm{C}$ with primary antibodies for VEGF and B7-2 (Santa Cruz Biotechnology, Santa Cruz, CA) at 1:250 and 1:1,000 dilutions, respectively. The primary antibody for Iba1 (Wako Chemicals, Neuss, Germany) was used at a $0.5 \mu \mathrm{g} /$ $\mathrm{ml}$ concentration. The goat anti-mouse (for VEGF and B7-2) and goat anti-rabbit (for Iba1) HRP secondary antibodies (Santa Cruz Biotechnology) were added at a 1:5,000 dilution for $1 \mathrm{hr}$ at room temperature, and the VEGF, B7-2, and Iba1 proteins were detected with the Amersham ECL Plus Western blotting detection reagent (GE Healthcare, EuroClone). Protein expression levels were then quantified in ImageJ.

\section{RNA Extraction, Gene Expression, and Analysis by RT-qPCR}

RNA was extracted from the C13NJ and BV-2 cell lines with the RNeasy Mini kit (Qiagen, Milan, Italy) and DNase treatment, according to the manufacturer's instructions. RNA was quantified with a NanoDrop 1000 UV spectrophotometer (NanoDrop Technologies, Wilmington, DE).

Reverse transcription was performed with $125 \mathrm{ng}$ total RNA in a final volume of $25 \mu$ containing $500 \mathrm{mM} \mathrm{KCl}$, $0.1 \mathrm{mM}$ EDTA, $100 \mathrm{mM}$ Tris- $\mathrm{HCl}$ ( $\mathrm{pH} 8.3$ ), $5.5 \mathrm{mM} \mathrm{MgCl}_{2}$, $500 \mu \mathrm{M}$ of each dNTP, $2.5 \mu \mathrm{M}$ random hexamers, $0.4 \mathrm{U} / \mu \mathrm{l}$ RNase inhibitor, and $1.25 \mathrm{U} / \mu \mathrm{l}$ Multiscribe reverse transcriptase from the TaqMan reverse transcription reagents kit (Life Technologies, Milan, Italy). The reverse transcription reaction was carried out at $25^{\circ} \mathrm{C}$ for $10 \mathrm{~min}, 48^{\circ} \mathrm{C}$ for $30 \mathrm{~min}$, and $95^{\circ} \mathrm{C}$ for $3 \mathrm{~min}$. For each sample, $12.5 \mathrm{ng}$ cDNA was added to $10 \mu \mathrm{l}$ of the PCR mix containing the dedicated primers/probe set
$(1 \times)$ and $1 \times$ Universal PCR Master Mix (Life Technologies). Specific exon-spanning TaqMan gene expression assays (Life Technologies) were used: VEGF Hs00900055_m1 for human VEGF, Hs01567026_m1 for human B7-2, Mm01281449_m1 for mouse B7-2, and Mm00444543_m1 for mouse B7-2. $18 \mathrm{~S}$ (Eukaryotic 18S rRNA Endogenous Control P.N. 4319413E; Life Technologies) was used as a housekeeping gene for both human and mouse cell lines. Samples were subjected to 40 cycles of amplification at $95^{\circ} \mathrm{C}$ for $15 \mathrm{sec}$ and $60^{\circ} \mathrm{C}$ for $60 \mathrm{sec}$ in the ABI Prism 7900 sequence detector (Life Technologies) in triplicate. Gene expression analysis was evaluated relative to the housekeeping gene transcript by using the comparative $\mathrm{Cq}$ method (Livak et al., 2001). To analyze gene expression levels between treatments within the two cell lines, a $t$-test was used, and a $P$ value of at least 0.05 was considered to be statistically significant. Transcript-level comparisons were performed in SPSS 17.0 (SPSS Inc., Chicago, IL).

\section{Detection of DNA Fragmentation To Evaluate Apoptosis}

To study apoptosis, DNA integrity in the BV-2/SHSY5Y and C13NJ/SHSY5Y cocultures was evaluated with the DNA fragmentation detection kit (FragEL fragmentation detection kit, Fluorescent-TdT Enzyme; Calbiochem, Life Technology). The principle of the Fluorescein-FragEL method is that terminal deoxynucleotidyl transferase (TdT) catalyzes the addition of fluorescein-labeled and unlabeled deoxynucleotides to the $3^{\prime}-\mathrm{OH}$ ends that are generated by endonucleases during apoptotic degeneration. SH-SY5Y/BV-2 and SH-SY5Y/ $\mathrm{C} 13 \mathrm{NJ}$ cocultures were plated on coverslips in their appropriate complete growth media; SH-SY5Y and BV-2 cells were seeded at densities of 100,000 and 10,000 cells, respectively, whereas SH-SY5Y and C13NJ cells were seeded at densities of 100,000 and 20,000, respectively. After $24 \mathrm{hr}$ of starvation, the cells were treated with different concentrations of oxaliplatin and/or OA-GcMAF for 24 or $48 \mathrm{hr}$. At the end of the treatments, the medium was removed, and the cells were washed twice with cold PBS. The cells were first fixed in $0.5 \%$ paraformaldehyde in PBS for $10 \mathrm{~min}$ and then permeabilized for $2 \mathrm{~min}$ in cold acetone at room temperature. After three washes in TBS, the slides were incubated in $1 \times \mathrm{TdT}$ equilibration buffer for 30 min at room temperature. Next, the slides were incubated in a staining solution consisting of $57 \mu$ Fluorescein-FragEL TdT labeling reaction mix and $3 \mu \mathrm{l}$ TdT enzyme solution for $1.5 \mathrm{hr}$ at $37^{\circ} \mathrm{C}$ in a humidified atmosphere. The cells were washed three times in TBS for $1 \mathrm{~min}$ at room temperature, and the slides were eventually mounted onto a microscope slide with a drop of mounting medium. Images were acquired with a motorized Leica DM6000 B microscope equipped with a DFC350FX camera (Leica, Mannheim, Germany) at $\times 200$, and the signal intensity was analyzed in ImageJ.

\section{RESULTS}

\section{Effects of Oxaliplatin and OA-GcMAF Treatment on the Viability and Density of BV-2 and C13NJ} cells

BV-2 and C13NJ microglial cell viability was evaluated by the WST-8 assay after $24 \mathrm{hr}$ of treatment with 
A

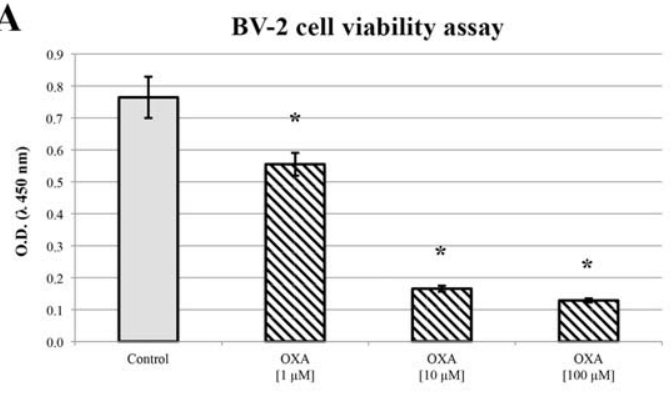

B

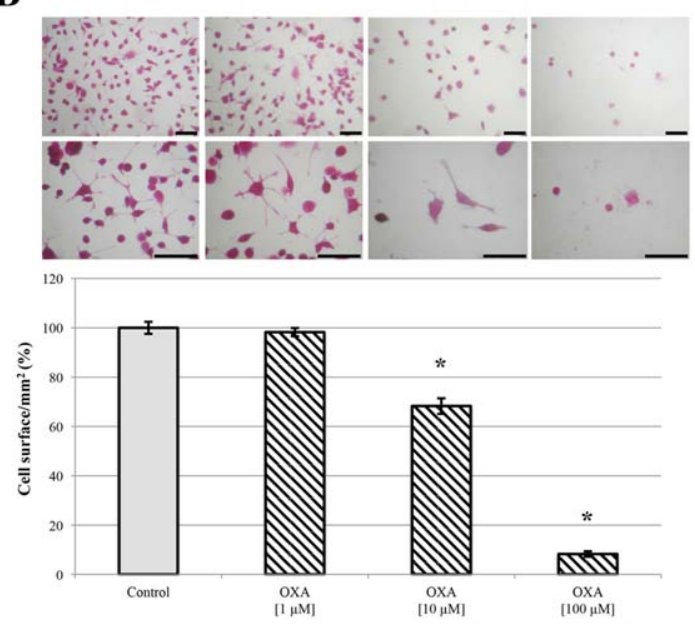

Fig. 1. Viability and density of BV-2 and C13NJ cells after treatment with oxaliplatin. A,C: Cell viability was evaluated by the WST-8 assay. Histograms show the decrease of BV-2 (A) and C13NJ (C) metabolic activity after $24 \mathrm{hr}$ of oxaliplatin treatment at different concentrations. B,D: Cell density was evaluated by light microscopy after $\mathrm{H} \& \mathrm{E}$ staining and quantified in ImageJ. Images show the decrease of cell density of BV-2 (B) and C13NJ (D) cells after $24 \mathrm{hr}$ of oxaliplatin

oxaliplatin at different concentrations. As shown in Figure $1 \mathrm{~A}, \mathrm{C}$, the viability of $\mathrm{BV}-2$ and $\mathrm{C} 13 \mathrm{NJ}$ cells decreased with increasing oxaliplatin concentration, as expected. Significant differences $(P<0.05)$ were observed when BV-2 cells were treated with oxaliplatin concentrations of 1,10 , and $100 \mu \mathrm{M}$ compared with untreated control cells (Fig. 1A). For C13NJ cell viability, significant differences $(P<0.05)$ were observed for oxaliplatin concentrations of $100 \mu \mathrm{M}, 500 \mu \mathrm{M}$, and $1 \mathrm{mM}$ compared with untreated control cells (Fig. 1C). These results confirm the known cytotoxicity of oxaliplatin and demonstrate that human microglial cells are somehow more resistant than murine cells to oxaliplatin-induced damage.

The significant decrease of oxaliplatin-induced cell viability in both microglial cell lines was associated with a significant decrease of cell density, as evaluated by light microscopy. Cell density variations were then quantified in the appropriate software and expressed as cell surface $/ \mathrm{mm}^{2}$. Consistent with the results obtained from the cell viability assay, BV-2 cell density was significantly decreased
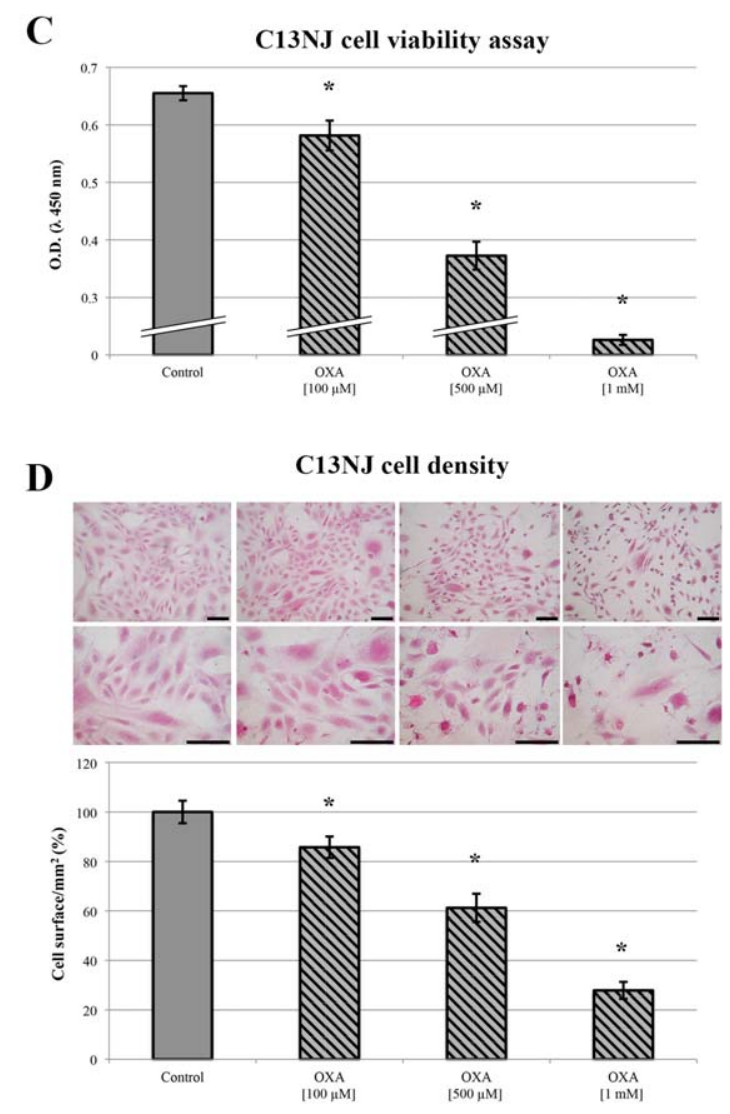

treatment at different concentrations. Histograms show the mean area occupied by BV-2 (B) and C13NJ (D) surfaces in five different fields $\left(1 \mathrm{~mm}^{2}\right)$ on each coverslip. Two coverslips were blindly counted for each experimental point. Results are mean \pm SEM for two replicates; each experiment was performed three times. O.D., optical density; OXA, oxaliplatin. ${ }^{\star} P<0.05$ vs. control. Scale bars $=100 \mu \mathrm{m}$.

$(P<0.05)$ when the cells were treated with 10 and 100 $\mu \mathrm{M}$ oxaliplatin, whereas no significant difference was observed for the lowest concentration of oxaliplatin (1 $\mu \mathrm{M}$; Fig. 1B). C13NJ cell density variations fully confirmed the data obtained from the cell viability assay, demonstrating that a significant decrease in cell density occurred for all oxaliplatin concentrations tested (Fig. 1D).

These results demonstrate that the decrease of total cell dehydrogenase activity was effectively the result of a decrease in the number of viable cells rather than a nonspecific decrease of cell metabolic activity. Furthermore, the data obtained both from the cell viability assay and from the morphological analysis indicated that the most appropriate concentration of oxaliplatin to induce reversible cell damage on microglial cells was $10 \mu \mathrm{M}$ for BV-2 cells and $100 \mu \mathrm{M}$ for $\mathrm{C} 13 \mathrm{NJ}$ cells. Therefore, these oxaliplatin concentrations were used in the experiments described below.

When the BV-2 and C13NJ microglial cells were treated for $48 \mathrm{hr}$ with OA-GcMAF (800 pM), a 
A

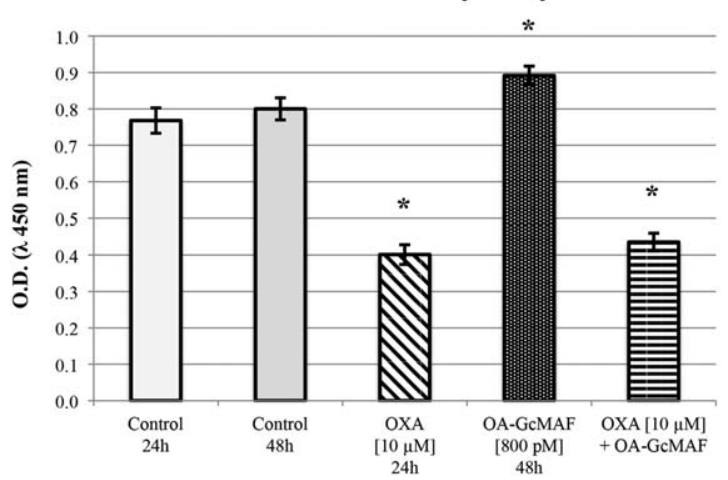

Fig. 2. Viability of BV-2 and C13NJ cells after treatment with oxaliplatin and OA-GcMAF. A,B: OA-GcMAF treatment of BV-2 and C13NJ cells for $48 \mathrm{hr}$ induces significant changes in cell viability. Oxaliplatin treatment, as expected, significantly decreases cell viability compared with controls. OA-GcMAF pretreatment for $24 \mathrm{hr}$ before
B

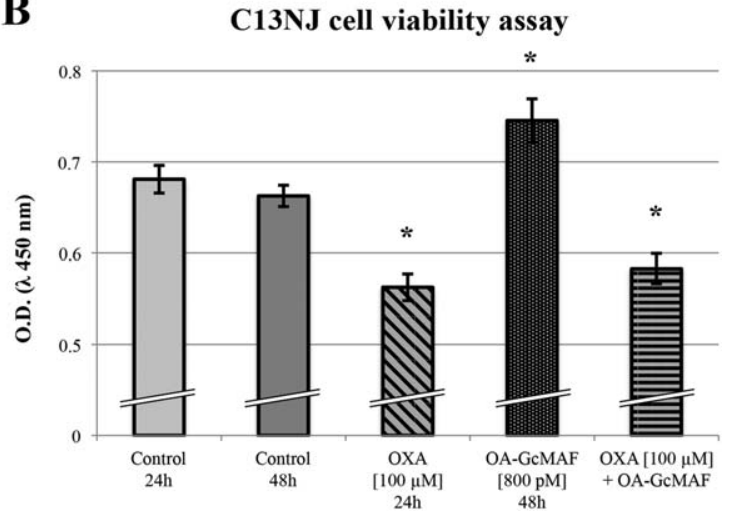

adding oxaliplatin does not significantly affect cell viability compared with oxaliplatin treatment alone. Results are mean \pm SEM for three replicates. Each experiment was performed three times. O.D., optical density; OXA, oxaliplatin; OA-GcMAF, oleic acid Gc-proteinderived macrophage-activating factor. ${ }^{\star} P<0.05$ vs. control.
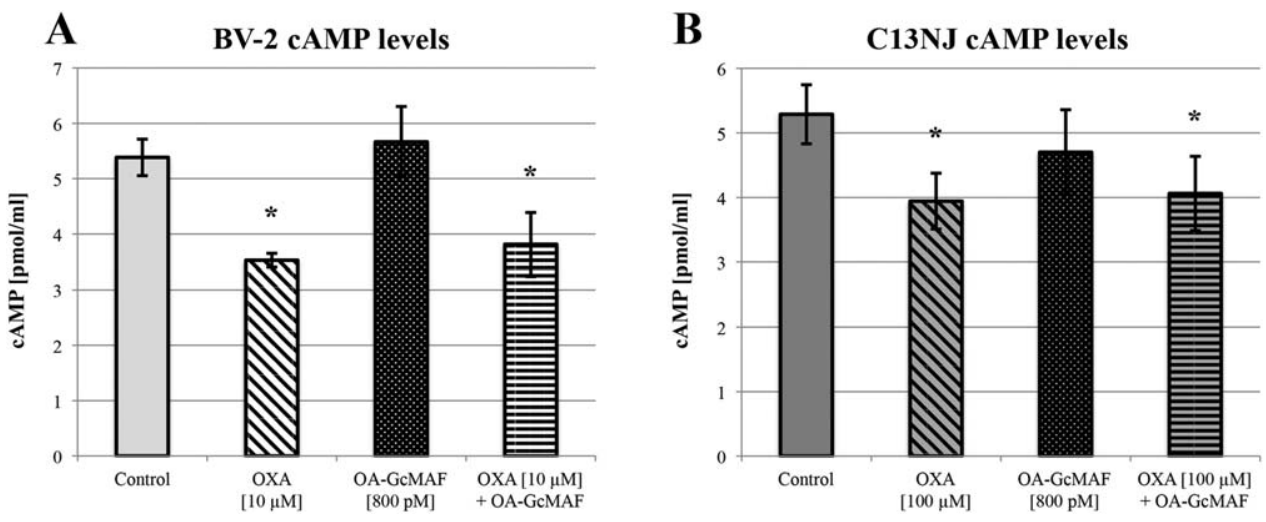

Fig. 3. cAMP levels from BV-2 and C13NJ cells after treatment with oxaliplatin and OA-GcMAF. A,B: cAMP levels, evaluated by ELISA assay, were measured after 25 min of 800 pM OA-GcMAF treatment, suggesting that OA-GcMAF does not activate the cAMP signal transduction pathway in $\mathrm{BV}-2$ or $\mathrm{C} 13 \mathrm{NJ}$ cells. In contrast, oxaliplatin induces a significant decrease of cAMP, which is not affected by the presence of OA-GcMAF. OXA, oxaliplatin; OA-GcMAF, oleic acid Gc-proteinderived macrophage-activating factor.

significant increase $(P<0.05)$ in cell viability was observed compared with control cells (untreated and cultured for the same time in the same media but without OA-GcMAF; Fig. 2A,B). However, the decrease in cell viability induced by oxaliplatin at the appropriate concentration $(10 \mu \mathrm{M}$ for $\mathrm{BV}-2$ and $100 \mu \mathrm{M}$ for $\mathrm{C} 13 \mathrm{NJ})$ was not reversed by pretreatment with OA-GcMAF. In this case, the presence of OA-GcMAF, added $24 \mathrm{hr}$ before the treatment with oxaliplatin and still present during the treatment with oxaliplatin, did not restore BV-2 or C13NJ cell viability (Fig. 2A,B). These results demonstrate that, despite their differential resistance to oxaliplatin-induced damage, the murine and the human cell lines show an analogous behavior to OA-GcMAF in terms of cell viability.

\section{Treatment of BV-2 and C13NJ Cells with Oxaliplatin and OA-GcMAF: Study of cAMP and VEGF Expression}

Oxaliplatin treatment of BV-2 $(10 \mu \mathrm{M})$ and $\mathrm{C} 13 \mathrm{NJ}$ $(100 \mu \mathrm{M})$ cells induced a significant decrease in cAMP levels after $25 \mathrm{~min}$ (Fig. 3A,B). These results were consistent with the damage inflicted by oxaliplatin and cell viability measurements. OA-GcMAF (800 pM) did not significantly affect microglial cAMP levels in BV-2 or C13NJ cells compared with untreated control cells (Fig. 3A,B). When BV-2 and C13NJ cells were treated with OA-GcMAF and then with oxaliplatin, no significant variations in cAMP levels were observed compared with the oxaliplatin-reduced cAMP levels (Fig. 3A,B). These results suggest that the cellular damage inflicted by 
$\mathbf{A}$

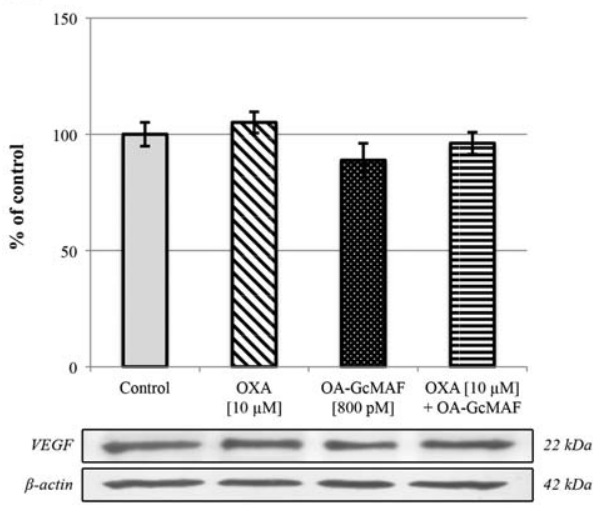

B BV-2 (VEGF mRNA expression)

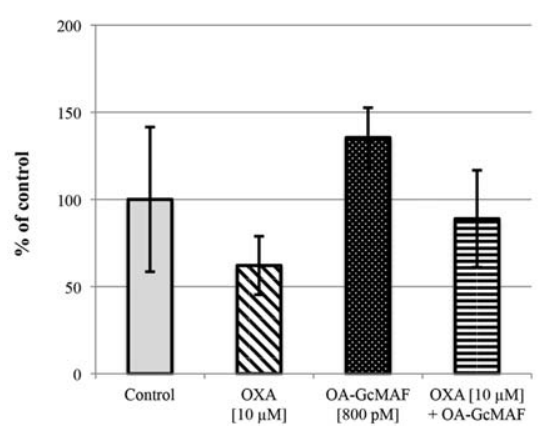

Fig. 4. VEGF expression in $\mathrm{BV}-2$ and $\mathrm{C} 13 \mathrm{NJ}$ cells after oxaliplatin and OA-GcMAF treatment. Western blot, densitometric analysis, and RT-qPCR. A,C: VEGF expression, evaluated by Western blot analysis and quantified in Image J, did not change after BV-2 and C13NJ cells were treated with 800 pM OA-GcMAF for $48 \mathrm{hr}$, with oxaliplatin alone for $24 \mathrm{hr}$, or with a combination of both stimuli. B,D: Evaluation of VEGF mRNA by RT-qPCR confirmed the data

oxaliplatin was not reversed by pretreatment with OAGcMAF, even at the level of cAMP second messenger formation.

In addition, because microglial cells produce VEGF, and considering the role of the cAMP/VEGF signaling pathway in microglia (Licht and Keshet, 2013; Krause et al., 2014), we investigated the variations of VEGF expression in BV-2 and in C13NJ microglial cells after treatment with oxaliplatin for $24 \mathrm{hr}$ and/or with OAGcMAF for $48 \mathrm{hr}$. The results showed that the treatment of BV-2 cells and C13NJ cells with 800 pM OA-GcMAF did not induce a significant change in VEGF expression, as demonstrated by Western blot and densitometric analysis (Fig. 4A,C). Quantitative evaluation by RT-qPCR confirmed that OA-GcMAF did not induce any significant modification in the levels of VEGF mRNA in BV-2 or in C13NJ cells (Fig. 4B,D). Furthermore, VEGF expression was not significantly modified by oxaliplatin treatment or a combination of oxaliplatin/OA-GcMAF in BV-2 or C13NJ cells compared with control cells. These results were further confirmed by RT-qPCR analysis (Fig. 4B,D). Taken together, these results indicate

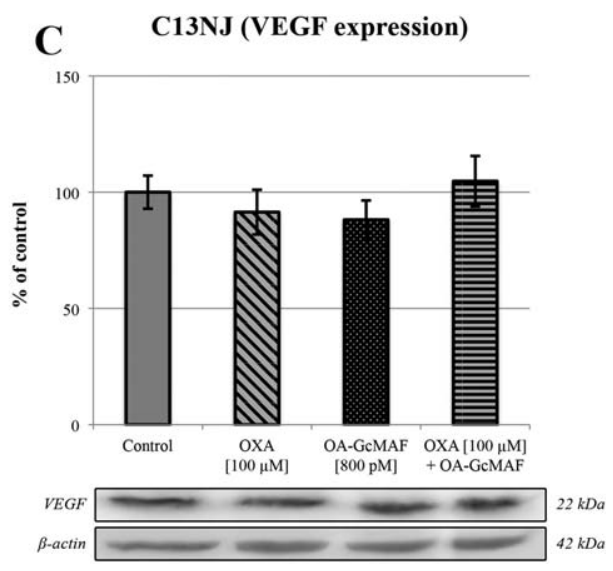

\section{C13NJ (VEGF mRNA expression)}

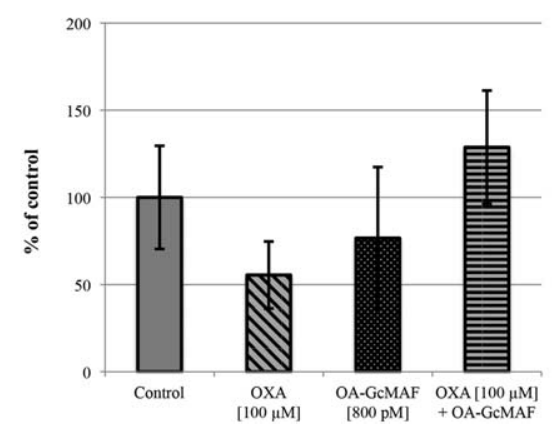

obtained by Western blot analysis and densitometric quantification. No significant differences are observed in BV-2 or C13NJ cells after OA-GcMAF and oxaliplatin treatment (alone or in combination). Results are mean \pm SEM for two replicates. Each experiment was performed three times. OXA, oxaliplatin; OA-GcMAF, oleic acid Gc-protein-derived macrophage activating factor.

that OA-GcMAF did not activate the cAMP/VEGF signaling pathway in BV-2 and C13NJ cells, nor did it counteract the oxaliplatin-induced effects. Therefore, these results are consistent with the cAMP levels in response to stimuli in both cell lines, confirming the observation that the cAMP/VEGF signal transduction pathway was not affected by oxaliplatin and/or OAGcMAF treatment.

\section{B7-2 Expression in BV-2 and C13NJ Cells Following Oxaliplatin and OA-GcMAF Treatment}

To assess whether the increase of cell viability induced by OA-GcMAF treatment was the result of microglial activation, we investigated the expression of B7-2, a protein that is upregulated in activated microglia and is involved in breaking the synaptic contacts of impaired neurons to allow nerve regeneration and neuritogenesis (De Simone et al., 1995). B7-2 expression was evaluated by Western blot analysis, and the images were densitometrically quantified in BV-2 and in C13NJ microglial cells after oxaliplatin and/or OA-GcMAF 

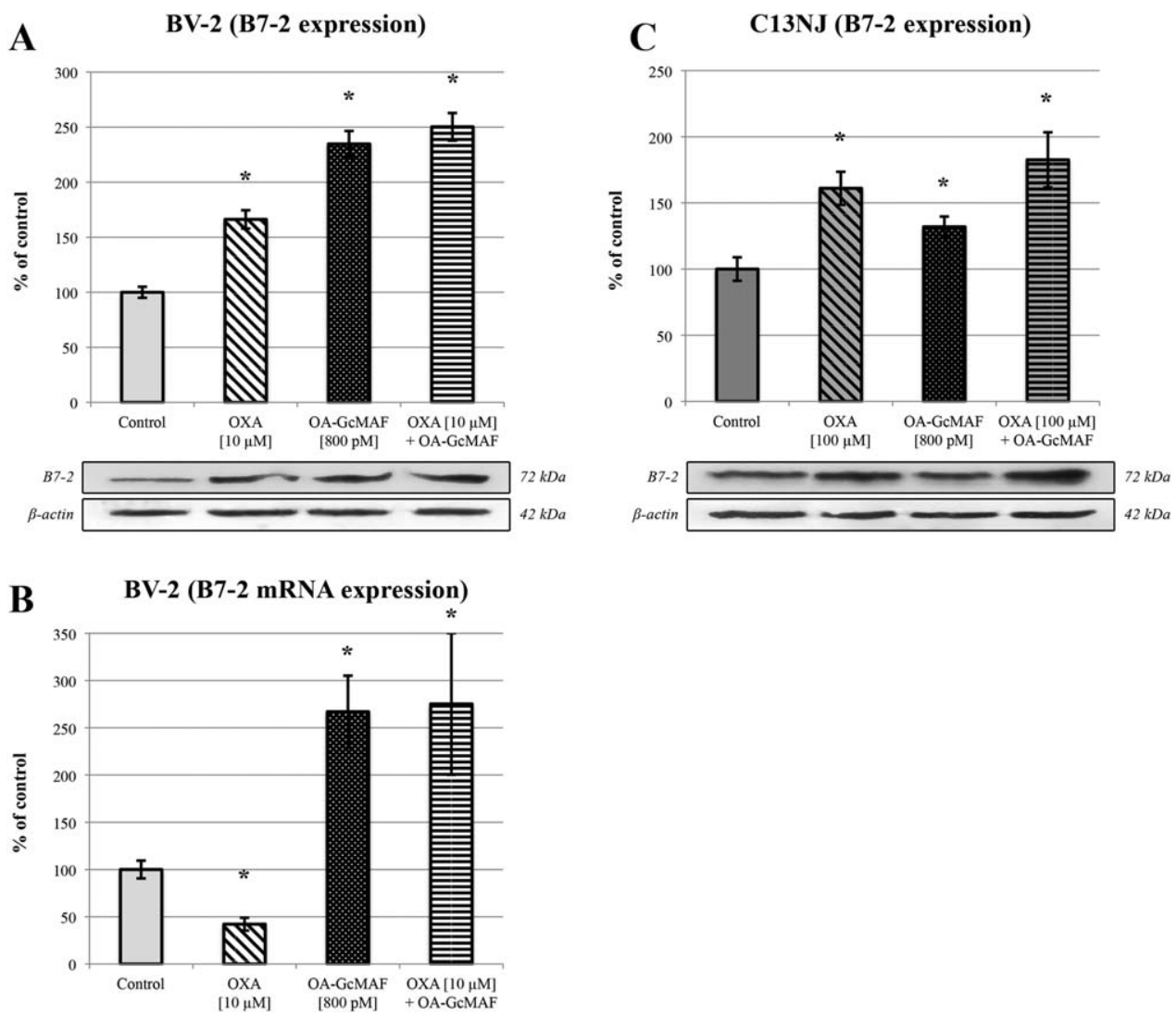

Fig. 5. B7-2 expression in BV-2 and C13NJ cells after oxaliplatin and OA-GcMAF treatment. Western blot, densitometric analysis, and RT-qPCR. A,C: After OA-GcMAF treatment, B7-2 expression, evaluated by Western blot analysis and quantified in ImageJ, was significantly increased compared with untreated control cells. Oxaliplatin treatment alone in BV-2 and $\mathrm{C} 13 \mathrm{NJ}$ cells induced, as expected, a significant increase of B7-2 expression, which is further increased when OA-GcMAF and oxaliplatin act together $\left({ }^{\star} P<0.05\right)$. B: Evaluation of B7-2 mRNA by RT-qPCR in BV-2 cells confirms the OA-

treatment. Oxaliplatin treatment of BV-2 and of C13NJ microglial cells at concentrations of $10 \mu \mathrm{M}$ and $100 \mu \mathrm{M}$, respectively, induced a significant increase in B7-2 expression in comparison with untreated control cells (Fig. 5A,C), as expected in the case of toxicants (Di Cesare Mannelli et al., 2013b). After $48 \mathrm{hr}$ of treatment with OA-GcMAF alone, the expression of B7-2 was significantly increased in both BV-2 and C13NJ microglial cells compared with untreated control cells (Fig. 5A,C). The expression of $\mathrm{B} 7-2$ in $\mathrm{BV}-2$ and $\mathrm{C} 13 \mathrm{NJ}$ cells was further increased with a combination of oxaliplatin and OA-GcMAF (Fig. 5A,C). However, it is notable that, although both oxaliplatin and OA-GcMAF induced an increase in B7-2 expression, the former is a known toxicant (as demonstrated in the literature and confirmed by the results from cell viability assay), whereas the latter stimulated cell viability in both murine and human cells. These results suggest that OA-GcMAF positively affects basal activation of microglial cells in the presence of a steady-state microenvironment. Furthermore, these data

GcMAF-induced increase in expression, both alone and in the presence of oxaliplatin. However, B7-2 mRNA and protein expression disagree when the cells are treated with oxaliplatin alone. Evaluation of B7-2 mRNA in C13NJ cells is not shown because no signal was detected by RT-qPCR. Results are mean \pm SEM for two replicates. Each experiment was performed three times. OXA, oxaliplatin; OAGcMAF, oleic acid Gc-protein-derived macrophage activating factor. $\star P<0.05$ vs. control.

indicate that OA-GcMAF could make the microglial cells more responsive to activation by a toxicant, i.e., oxaliplatin. We hypothesize that the damage inflicted by oxaliplatin leads to a strong microglial activation in the presence of OA-GcMAF, in turn leading to faster clearance of dying cells and cellular debris. Evaluation of B72 mRNA by RT-qPCR in BV-2 cells provided some interesting information (Fig. 5B). In the presence of OAGcMAF (alone or in combination with oxaliplatin), BV2 cells expressed higher levels of B7-2 mRNA compared with untreated control cells. This result confirms the data obtained from Western blot analysis and densitometry (Fig. 5A). However, when BV-2 cells were treated with oxaliplatin alone, the level of B7-2 mRNA was significantly lower compared with the control cells, whereas the level of B7-2 protein was higher (Fig. 5A). This apparently odd result can be explained because oxaliplatin interacts not only with DNA but also with mRNA and possibly interferes with its integrity (Becker et al., 2014). 
A

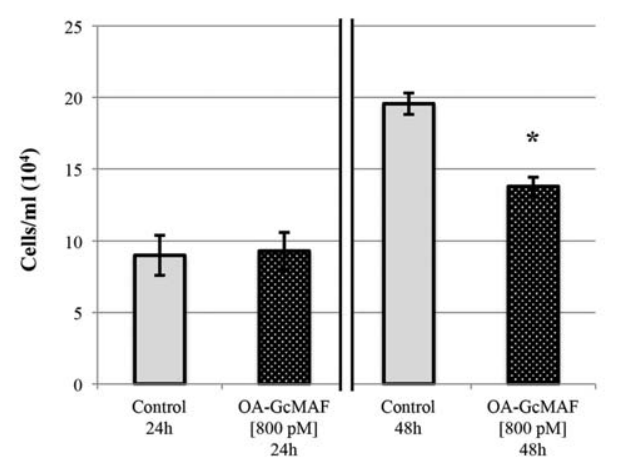

B

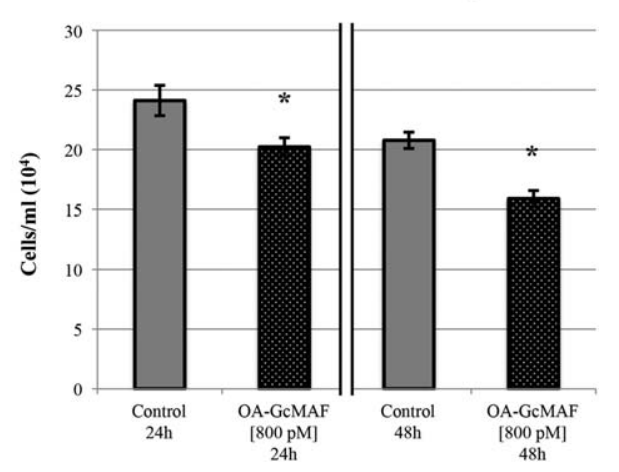

Fig. 6. BV-2 and C13NJ cell counts after OA-GcMAF treatment. A,B: BV-2 and C13NJ cell counts by standard trypan blue staining. OA-GcMAF treatment for 24 and $48 \mathrm{hr}$ is not associated with an increase in the number of cells compared with controls. Results are mean \pm SEM for five replicates. Each experiment was performed three times. OA-GcMAF, oleic acid Gc-protein-derived macrophage activating factor. ${ }^{\star} P<0.05$ vs. control.

RT-qPCR evaluation in C13NJ cells showed undetectable expression of B7-2 mRNA at all of the experimental points (data not shown). This result is confirmed by data available in the literature demonstrating that the expressions of the B7-2 protein and its mRNA are unrelated (The Human Protein Atlas; http://www.proteinatlas.org/ENSG00000114013-CD86/tissue).

To rule out the possibility that microglial activation induced by OA-GcMAF could result in an increase of microglial cells, a potentially detrimental phenomenon, cell counts were performed with standard trypan blue staining 24 and $48 \mathrm{hr}$ after OA-GcMAF treatment. As shown in Figure 6A, there was no significant increase in cell numbers when BV-2 cells were treated with OAGcMAF for $24 \mathrm{hr}$. Conversely, after $48 \mathrm{hr}$ of OAGcMAF treatment, a significant decrease in BV-2 cell numbers was observed (Fig. 6A). OA-GcMAF treatment of C13NJ cells for 24 or $48 \mathrm{hr}$ resulted in a significant decrease in cell numbers (Fig. 6B). These results suggest that OA-GcMAF could increase microglial activation (with higher expression of B7-2 protein) without leading to a concomitant and potentially harmful increase in cell numbers. These results also confirm that the behavior of murine and human microglial cells, in terms of activation, is essentially the same, albeit not identical.

\section{Morphology and Apoptosis of BV-2/SH-SY5Y and C13NJ/SH-SY5Y Cocultures Following Oxaliplatin and OA-GcMAF Treatment}

Data from cocultures of murine microglial cells and human neurons (BV-2/SH-SY5Y) show that the morphology and the interactions between neurons and microglial cells were deeply affected by $10 \mu \mathrm{M}$ oxaliplatin treatment. As shown in Figure 7A, microglia-neuron connections and neuron density were decreased after oxaliplatin treatment (Fig. 7Ab) compared with control (Fig. 7Aa) and OA-GcMAF-treated cells (Fig. 7Ac). After oxaliplatin treatment, debris (Fig. 7A, white arrows) and numerous microglial cells (Fig. 7A, black arrowheads) were closely contacting and surrounding the neurons (Fig. 7Ab), suggesting a specific response of microglia to the damage induced by the oxaliplatin-altered microenvironment (Wake et al., 2009). Microglia-neuron connections (Fig. 7A, white arrowheads) and the regular distribution of glia around the neurons were restored when the cocultures were pretreated with OA-GcMAF for $24 \mathrm{hr}$ prior to oxaliplatin addition (Fig. 7Ad). However, the neuron density was still reduced because of the toxic effect of oxaliplatin, and a large amount of debris could still be detected around the cells.

To investigate and quantify the cell damage inflicted by oxaliplatin and the protective effect of OA-GcMAF further, a TUNEL assay was performed to analyze apoptosis. As shown in Figure 7B, very few apoptotic cells were detectable in control cells (Fig. 7Ba) or in cells treated with OA-GcMAF (Fig. 7Bc), as expected for cell "healthy" cultures. However, when the cells were treated with $10 \mu \mathrm{M}$ oxaliplatin (Fig. 7Bb), the number of apoptotic cells significantly increased; conversely, and in line with the protective effect of OA-GcMAF, the number of apoptotic cells was significantly reduced when the cocultures were pretreated with OA-GcMAF for $24 \mathrm{hr}$ before adding oxaliplatin (Fig. 7Bd). Variations in apoptosis, as observed by fluorescence microscopy, were then quantified in the appropriate software and expressed as cell surface $/ \mathrm{mm}^{2}$ (Fig. 7C). As shown by histograms, taking the level of apoptosis in control cells to be $100 \%$, oxaliplatin increased the level of apoptosis by more than threefold. When the cocultures were pretreated with OA-GcMAF and then with oxaliplatin, the level of apoptosis was significantly decreased compared with oxaliplatin treatment alone.

Additionally, the data obtained from cocultures of human microglial cells and human neurons (C13NJ/ SH-SY5Y) demonstrated that the cell morphology and 
A

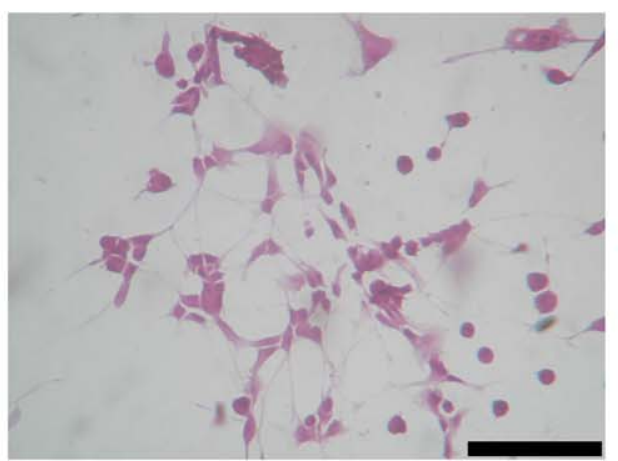

a

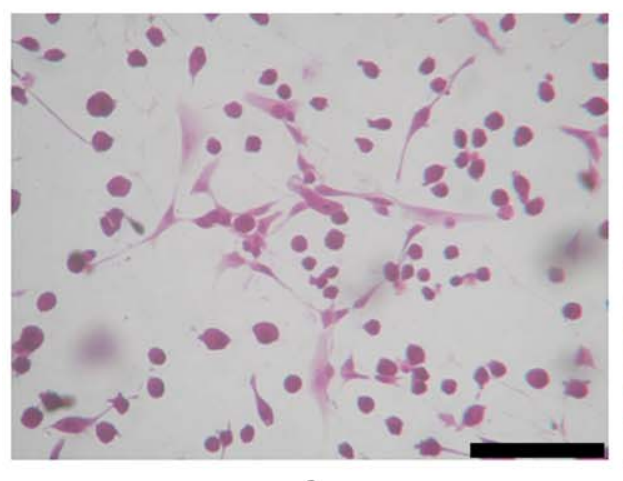

c
BV-2 / SH-SY5Y co-culture

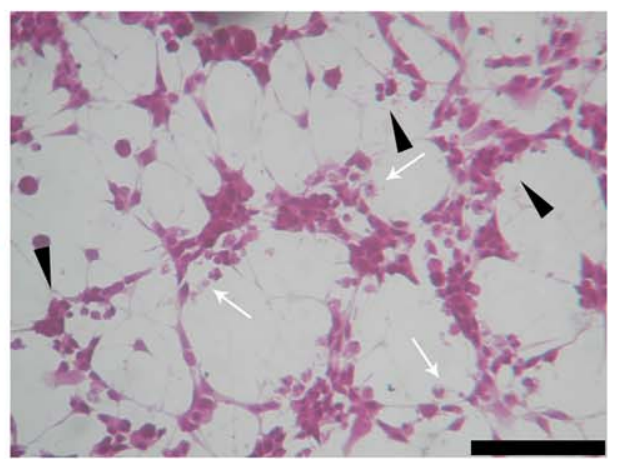

b

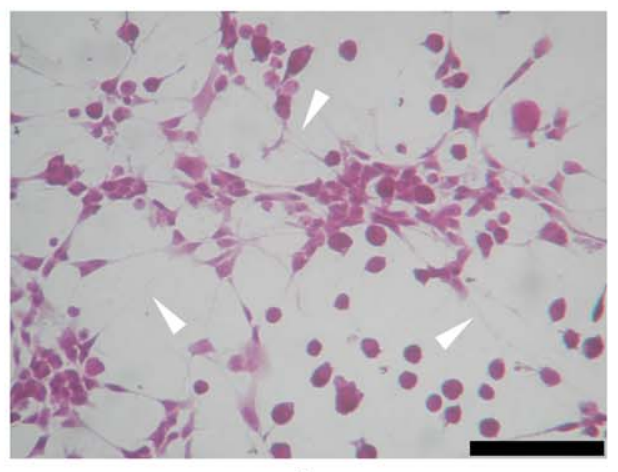

d
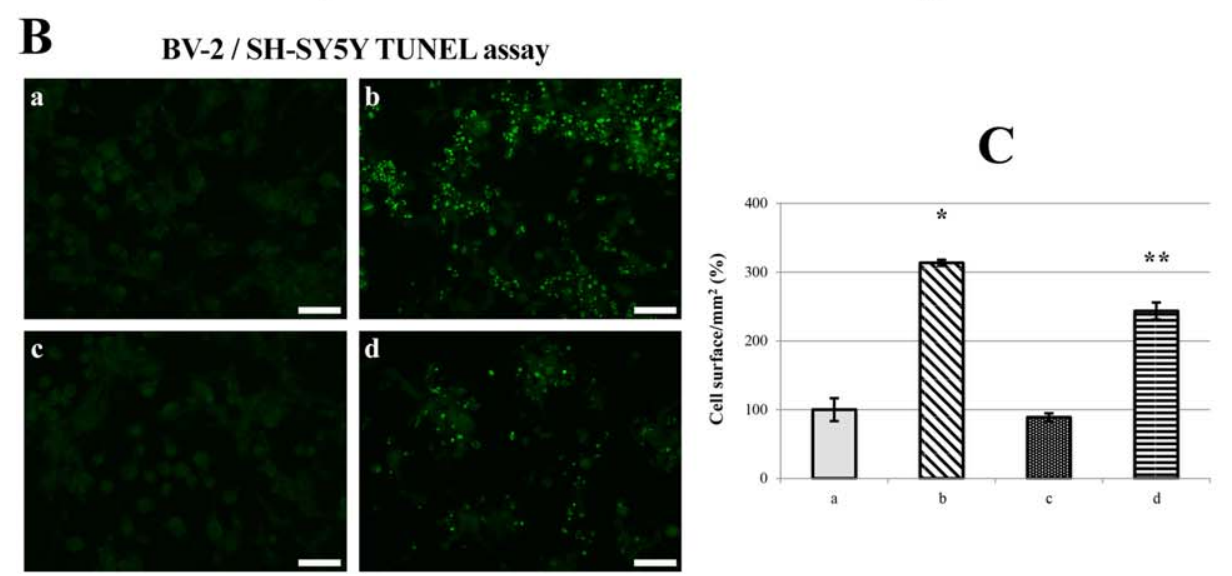

Fig. 7. BV-2/SH-SY5Y coculture morphology and apoptosis after oxaliplatin and OA-GcMAF treatment. A: Cell morphology. a: Untreated control cells. Neurons show a typical triangular body, evident nucleus, wide cytoplasm, and several long cytoplasmic elongations. Microglia are represented primarily by deeply stained rounded cells with thin elongations. Elongations arising from the glial cells and neurons form an intricate net linking the microglia and neurons to each other. b: Ten micromolar oxaliplatin-treated cells. Most of the cellular connections are deeply damaged, and only a few neurons are present. Much debris (white arrows), a clear sign of cell degeneration, is present. Numerous glial cells are distributed along and around the few damaged neurons (black arrowheads). c: OA-GcMAF-treated cells. No significant differences are observed in the microglia or neurons when the coculture is treated with OA-GcMAF compared with untreated control cells. No debris or clusters of damaged cells can be observed. Numerous cell connections are present. d: OA-GcMAF and oxaliplatin-treated cells. Proper connections between neurons and glia (white arrowheads) are restored compared with oxaliplatin treatment alone. Neuron density remains decreased because of the toxic effects of oxaliplatin. B: TUNEL assay. In control cells (a) as well as in cells treated with OA-GcMAF (c), only a basal, low level of apoptosis is detectable. When the cells are treated with $10 \mu \mathrm{M}$ oxaliplatin (b), a significant increase in the number of apoptotic cells is observed; apoptosis is significantly reduced when cocultures are pretreated with OA-GcMAF for $24 \mathrm{hr}$ before adding oxaliplatin (d). In this case, only a few clusters of damaged cells are still present. $\times 200$. Variations of apoptosis were quantified in the appropriate software and expressed as cell surface $/ \mathrm{mm}^{2}(\mathbf{C})$. Results are mean \pm SEM for five replicates. Each experiment was performed three times. ${ }^{\star} P<0.05$ vs. control; $\star \star P<0.05$ vs. oxaliplatin. Scale bars $=100 \mu \mathrm{m}$. 


\section{A}

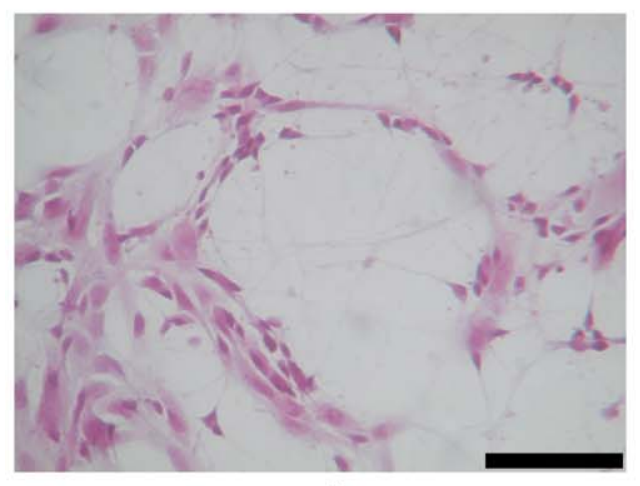

a

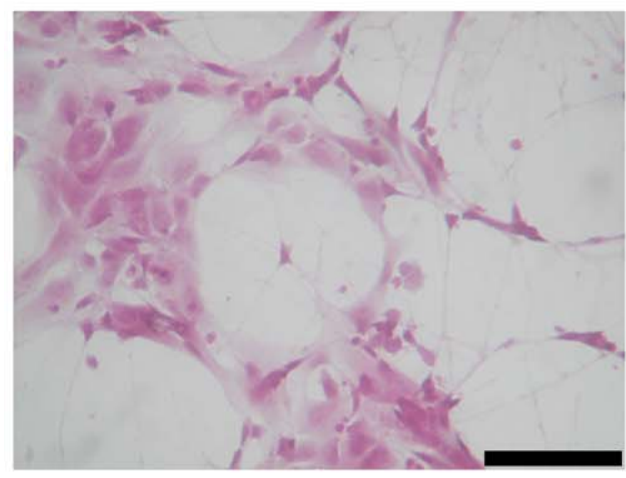

c
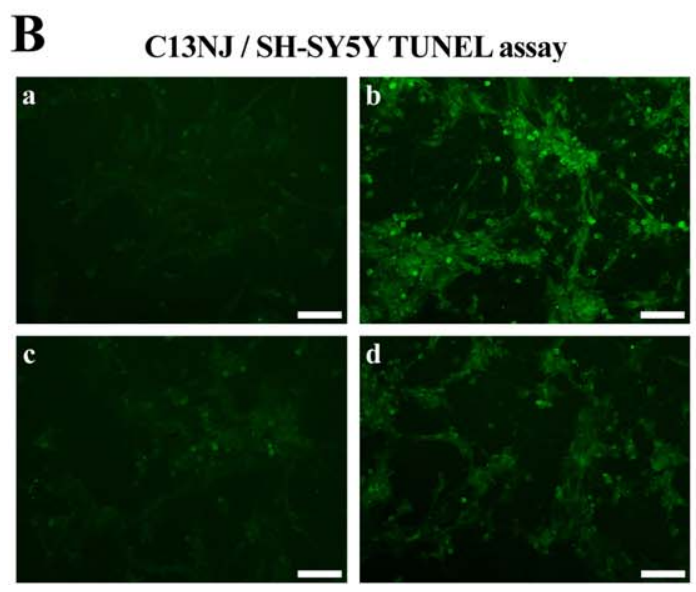

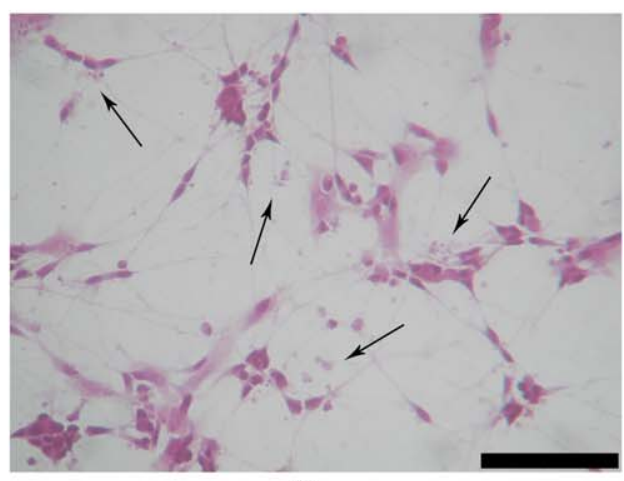

b

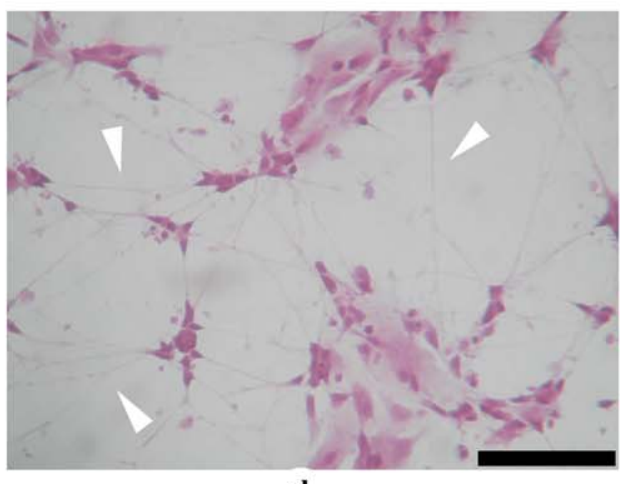

d
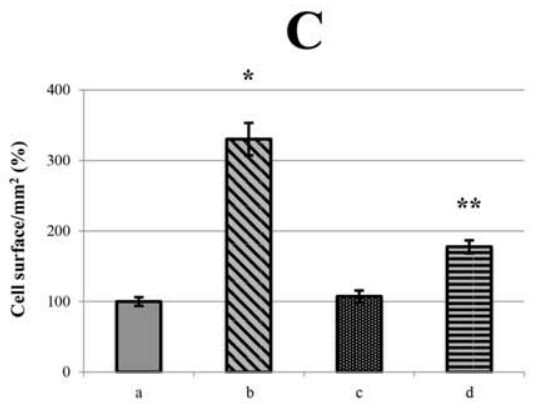

Fig. 8. C13NJ/SH-SY5Y coculture morphology and apoptosis after oxaliplatin and OA-GcMAF treatment. A: Cell morphology. a: Untreated control cells. Neurons show a typical triangular body, evident nucleus, wide cytoplasm, and several long cytoplasmic elongations. Microglia are represented primarily by deeply stained cells with different shape and size, sometimes forming clusters. Elongations arising from the glial cells and neurons form an intricate net linking the microglia and neurons to each other. b: Ten micromolar oxaliplatintreated cells. Most of the cellular connections are lost or deeply damaged, and only a few neurons are present. Much debris (black arrows), a clear sign of cell degeneration, is present. c: OA-GcMAF-treated cells. No significant differences are observed in the microglia or neurons when the coculture is treated with OA-GcMAF compared with untreated control cells. Numerous and well-defined cell connections are present. d: OA-GcMAF- and oxaliplatin-treated cells. Cell connections are increased (white arrowheads) compared with oxaliplatin treatment alone. Neuron density remains decreased because of the toxic effects of oxaliplatin, and some debris is still present. B: TUNEL assay. In control cells (a) as well as in cells treated with OA-GcMAF (c), only a basal, low level of apoptosis is detectable. When the cells are treated with $10 \mu \mathrm{M}$ oxaliplatin (b), a significant increase in the number of apoptotic cells is observed; cell death is significantly reduced when the cocultures are pretreated with OA-GcMAF for 24 hr before adding oxaliplatin (d). $\times 200$. Variations of apoptosis were quantified in the appropriate software and expressed as cell surface/ $\mathrm{mm}^{2}(\mathbf{C})$. Results are mean \pm SEM for five replicates. Each experiment was performed three times. ${ }^{\star} P<0.05$ vs. control; ${ }^{\star}{ }^{\star} P<0.05$ vs. oxaliplatin. Scale bars $=100 \mu \mathrm{m}$. 
interactions between neurons and microglial cells were deeply damaged by the treatment with oxaliplatin. Similarly to the previous results, OA-GcMAF was able to reverse the damage inflicted by oxaliplatin (Fig. 8A). Although the oxaliplatin concentration used for all the experiments involving the $\mathrm{C} 13 \mathrm{NJ}$ cells was $100 \mu \mathrm{M}$, a 10 $\mu \mathrm{M}$ concentration was used for the coculture experiments because the neurons are more sensitive to oxaliplatin than C13NJ cells.

We also observed a decrease in microglia-neuron connections in $\mathrm{C} 13 \mathrm{NJ} / \mathrm{SH}-\mathrm{SY} 5 \mathrm{Y}$ cocultures after oxaliplatin treatment (Fig. 8Ab). Many cells (both neurons and microglia) showed severe morphological modifications and large amounts of debris (Fig. 8A, black arrows) around the cells compared with the untreated control cells (Fig. 8Aa). OA-GcMAF treatment for $24 \mathrm{hr}$ prior to adding oxaliplatin restored the normal microglia-neuron connections (Fig. 8A, white arrowheads) and partially restored the cell morphology (Fig. 8Ad). Treatment with OA-GcMAF alone (Fig. 8Ac) did not induce significant changes compared with the control cells.

Apoptosis was evaluated by TUNEL assay also on $\mathrm{C} 13 \mathrm{NJ} / \mathrm{SH}-\mathrm{SY} 5 \mathrm{Y}$ cocultures. As shown in Figure 8B, only a few apoptotic cells were detectable in control cells (Fig. 8Ba) or cells treated with OA-GcMAF (Fig. 8Bc). When the cocultures were treated with $10 \mu \mathrm{M}$ oxaliplatin (Fig. 8Bb), a significant increase in the number of apoptotic cells was observed. However, apoptosis was significantly reduced when the cocultures were pretreated with OA-GcMAF for $24 \mathrm{hr}$ before adding oxaliplatin (Fig. $8 \mathrm{Bd}$ ). Variations of apoptosis, observed by fluorescent microscopy, were then quantified in the appropriate software and expressed as cell surface $/ \mathrm{mm}^{2}$ (Fig. 8C). As shown by histograms, an approximately threefold increase in apoptosis was observed after oxaliplatin treatment compared with control cells. When the cocultures were pretreated with OA-GcMAF and then with oxaliplatin, the level of apoptosis significantly decreased $(P<0.05)$ compared with oxaliplatin alone. Taken together, these results confirm that both the oxaliplatin-induced damage and the protective effects of OA-GcMAF were essentially the same in murine microglia/human neuron and human microglia/human neuron cocultures.

\section{DISCUSSION}

Several microglial cell lines are available and are currently used for research studies in addition to primary microglia. Microglial cultures were first described early in the 1930s, but the use of cultures to study microglial function occurred only after methods for obtaining and culturing large amounts of microglial cells had been developed and improved. The increased yield and homogeneity of cultured cells allows for increased data output in a shorter time compared with in vivo experiments. Furthermore, in vitro cultures present a beneficial tool for studying the activation state, releasable factors, and other crucial components that characterize microglia, which cannot be sufficiently examined in vivo. Among the different models of microglial cell lines used to study neuroinflammatory and neurodegenerative diseases are immortalized microglial cell cultures from human, rat, and mouse that are retrovirally or nonretrovirally transformed. Some of these culture models share relevant similarities, but at the same time they are separated by significant differences, depending on their origin, methods of culturing, functional aspects, and availability. One of the most extensively used microglia models is the retrovirally immortalized murine BV-2 cell line. This cell line is currently available on the market, is easily cultured, and is morphologically well characterized. However, the main criticism regarding BV-2 cells is that the cell line has been immortalized from mouse cortex and represents a cell line that is appropriate for murine rather than human studies. Therefore, there are also human cell lines because humans are one of the most important models. Some human immortalized microglial cell lines exist, among them HMO6 and C13NJ cells. Even if the characteristics of these cell lines make them appropriate for investigating human inflammatory processes and neurodegenerative diseases, they are rarely used because of the difficulty in obtaining and culturing them and the fact that little is known about their morphological properties. This study demonstrates that human $\mathrm{C} 13 \mathrm{NJ}$ and rat BV-2 microglia cell lines share common signaling mechanisms when treated with a neurotoxic chemotherapeutic agent, oxaliplatin, and with OA-GcMAF, a molecule able to reverse oxaliplatininduced neuronal damage and induce beneficial microglia activation.

At variance with its effects on neurons (Morucci et al., 2014), OA-GcMAF does not appear to restore the decrease of cell viability induced by oxaliplatin, and, consistently, it does not increase cAMP or induce the expression of VEGF in BV-2 or C13NJ cells. This study also demonstrates that the expression of $\mathrm{B} 7-2$, a specific protein marker of microglial cell activation, increases in BV2 and C13NJ cells in the presence of oxaliplatin-induced cell damage as well as after OA-GcMAF treatment alone. When BV-2 and C13NJ cells were triggered with oxaliplatin in the presence of OA-GcMAF, the expression of B7-2 protein further increased. It is noteworthy that OAGcMAF treatment was associated with an increase in cell viability as well as an increased expression of B7-2 protein without a concomitant increase of the cell number, lending credit to the hypothesis that OA-GcMAF-stimulated microglial cells contribute to overall neuroprotective effects by scavenging oxaliplatin-induced damage without nonspecific gliosis. This hypothesis is consistent with the notion that microglial cell activation is directly engaged in processes involved in restoring normal signaling and protecting against neurotoxicity (Milligan and Watkins, 2009).

Cocultures are a dynamic system for studying microglial interaction with neurons because microglia are able to establish wide-ranging interactions with the microenvironment. In agreement with these notions and considering the active role of microglia in preserving neuronal function and in restoring and/or preserving 
neuronal viability, we evaluated the interactions of mouse and human microglia with human neurons. Data from BV-2/SH-SY5Y as well as from C13NJ/SH-SY5Y cocultures showed that cellular morphology, interactions between neurons and microglial cells, and level of apoptosis were deeply affected by oxaliplatin treatment. The presence of OA-GcMAF significantly reduced the level of oxaliplatin-induced morphological alterations and apoptosis in both cocultures, leading us to hypothesize an active role for OA-GcMAF in counteracting the toxic effects of oxaliplatin on neurons through microglial activation.

We observed an interesting RT-qPCR result from our data, even though it was not strictly related to the target of the present study. In fact, although VEGF expression in microglia cells evaluated by Western blotting and densitometric analysis was superimposable to the expression of VEGF mRNA measured by RT-qPCR, the results for the B7-2 marker were somewhat unexpected. In this case, the expression of the B7-2 protein in BV-2 cells was only partially confirmed by RT-qPCR, and in C13NJ cells no B7-2 mRNA was detected. These results can be explained by considering that most reports on mRNA and protein find only a weak correlation between the abundances of these two classes of biological molecules (Maier et al., 2009). The correlation between mRNA and protein abundance depends on various biological and technical factors. Transcription and translation processes, mRNA decay, micro-RNAs, and a longer half-life of proteins are only some of the factors involved in this lack of correlation, supporting the fact that mRNA levels cannot be used to evaluate and predict the corresponding protein levels (Wang et al., 2008). In conclusion, because the BV-2 and the $\mathrm{C} 13 \mathrm{NJ}$ cell lines (alone and cocultured with human neurons) show analogous responses to oxaliplatin and OA-GcMAF in terms of cAMP/VEGF signal transduction pathway, cell viability, activation, and protein expression, we hypothesize that the murine BV-2 cell line is an excellent and validated experimental model for human microglial cells that is inexpensive and easy to culture and retrieve.

\section{ACKNOWLEDGMENTS}

The authors thank Dr. Jean Mazella (Institut de Pharmacologie Moléculaire et Cellulaire, Centre National de la Recherche Scientifique, Université de Nice-Sophia Antipolis, Valbonne, France) for kindly donating the C13NJ cell line and Immuno Biotech Ltd. (St. Peter Port, Guernsey, Channel Islands) for kindly donating OA-GcMAF.

\section{CONFLICT OF INTEREST STATEMENT}

$\mathrm{MR}$ is the consulting scientific director of Immuno Biotech Ltd. (the company isolating and purifying the GcMAF protein and producing OA-GcMAF). However, MR had no prior knowledge of the experimental protocols and did not participate in the actual planning of individual experiments. He participated in the analysis of the results only after they had been verified and validated by the other authors.

\section{REFERENCES}

Becker JP, Weiss J, Theile D. 2014. Cisplatin, oxaliplatin, and carboplatin unequally inhibit in vitro mRNA translation. Toxicol Lett 225:43-47.

Bignami E, Eng LF, Dahl D, Uyeda CT. 1972. Localization of the glial fibrillary acidic protein in astrocytes by immunoflourescence. Brain Res 43:429-435.

Blasi E, Mathieson BJ, Varesio L, Cleveland PA, Borchert PA, Rapp UR. 1985. Selective immortalization of murine macrophages from fresh bone marrow by a raf/myc recombinant murine retrovirus. Nature 318: $667-670$.

Blasi E, Barluzi R, Bocchini V, Mazolla R, Bistoni F. 1990. Immortalization of murine microglia cells by a v-raf/v-myc carrying retrovirus. J Neuroimmunol 27:229-237.

De Simone R, Giampaolo A, Giometto B, Gallo P, Levi G, Peschle C, Aloisi F. 1995. The costimulatory molecule B7 is expressed on human microglia in culture and in multiple sclerosis acute lesions. J Neuropathol Exp Neurol 54:175-187.

Di Cesare Mannelli L, Pacini A, Bonaccini L, Zanardelli M, Mello T, Ghelardini C. 2013a. Morphologic features and glial activation in rat oxaliplatin-dependent neuropathic pain. J Pain 14:1585-1600.

Di Cesare Mannelli L, Zanardelli M, Failli P, Ghelardini C. 2013b. Oxaliplatin-induced oxidative stress in nervous system-derived cellular models: could it correlate with in vivo neuropathy? Free Radic Biol Med 61:143-150.

Du BY, Song W, Bai L, Shen Y, Miao SY, Wang LF. 2012. Synergistic effects of combination treatment with bortezomib and doxorubicin in human neuroblastoma cell lines. Chemotherapy 58:44-51.

Griffiths M, Neal JW, Gasque P. 2007. Innate immunity and protective neuroinflammation: new emphasis on the role of neuroimmune regulatory proteins. Int Rev Neurobiol 82:29-55.

Henn A, Lund S, Hedtjarn M, Schrattenholz A, Porzgen P, Leist M. 2009. The suitability of BV2 cells as alternative model system for primary microglia cultures or for animal experiments examining brain inflammation. ALTEX 26:83-94.

Horvath R, McMenemy N, Alkaitis M, DeLeo J. 2008. Differential migration, LPS-induced cytokine, chemokine, and NO expression in immortalized BV-2 and HAPI cell lines and primary microglial cultures. J Neurochem 107:557-569.

Knyazeva EL, Grishchenko VM, Fadeev RS, Akatov VS, Permyakov SE, Permyakov EA. 2008. Who is Mr. HAMLET? Interaction of human alphalactalbumin with monomeric oleic acid. Biochemistry 47:13127-13137.

Kopec K, Caroll R. 1998. Alzheimer's $\beta$-amyloid peptide 1-42 induces a phagocytic response in murine microglia. J Neurochem 71:2123-2131.

Kovalevich J, Langford D. 2013. Considerations for the use of SH-SY5Y neuroblastoma cells in neurobiology. Methods Mol Biol 1078:9-21.

Krause TA, Alex AF, Engel DR, Kurts C, Eter N. 2014. VEGF production by CCR2-dependent macrophages contributes to laser-induced choroidal neovascularization. PLoS One 9:e94313.

Licht T, Keshet E. 2013. Delineating multiple functions of VEGF-A in the adult brain. Cell Mol Life Sci 70:1727-1737.

Livak KJ, Schmittgen TD. 2001. Analysis of relative gene expression data using real time quantitative PCR and the $2^{-\Delta \Delta \mathrm{Ct}}$ methods. Methods 25: 402-408.

Maier T, Güell M, Serrano L. 2009. Correlation of mRNA and protein in complex biological samples. FEBS Lett 583:3966-3973.

Marchand F, Perretti M, McMahon SB. 2005. Role of the immune system in chronic pain. Nat Rev Neurosci 6:521-532.

Milligan ED, Watkins LR. 2009. Pathological and protective roles of glia in chronic pain. Nat Rev Neurosci 10:23-36.

Morucci G, Branca JJV, Gulisano M, Ruggiero M, Paternostro F, Pacini A, Di Cesare Mannelli L, Pacini S. 2014. Gc-protein derived 
macrophage activating factor counteracts the neuronal damage induced by oxaliplatin. Anticancer Drugs 26:197-209.

Nguyen MD, Julien JP, Rivest S. 2002. Innate immunity: the missing link in neuroprotection and neurodegeneration? Nat Rev Neurosci 3: 216-227.

Peudenier S, Héry C, Montagnier L, Tardieu M. 1991. Human microglial cells: characterization in cerebral tissue and in primary cultures and study of their susceptibility to HIV-I infection. Ann Neurol 29:152161.

Romero-Sandoval EA, Horvath RJ, DeLeo JA. 2008. Neuroimmune interactions and pain: focus on glial-modulating targets. Curr Opin Investig Drugs 9:726-734.

Sahu U, Sidhar H, Ghate PS, Advirao GM, Raghavan SC, Giri RK. 2013. A novel anticancer agent, 8-methoxypyrimido[4',5': $4,5]$ thieno(2,3-b) quinoline-4(3H)-one induces neuro 2a neuroblastoma cell death through p53-dependent, caspase-dependent and -independent apoptotic pathways. PLoS One 8:e66430.
Thapa A, Vernon BC, De la Peña K, Soliz G, Moreno HA, López GP, Chi EY. 2013. Membrane-mediated neuroprotection by curcumin from amyloid- $\beta$-peptide-induced toxicity. Langmuir 29:11713-11723.

The human protein atlas. http://www.proteinatlas.org/ENSG00000114013CD86/tissue.

Torres-Platas SG, Comeau S, Rachalski A, Bo GD, Cruceanu C, Turecki G, Giros B, Mechawar N. 2014. Morphometric characterization of microglial phenotypes in human cerebral cortex. J Neuroinflammation 21:11:12.

Wake H, Moorhouse AJ, Jinno S, Kohsaka S, Nabekura J. 2009. Resting microglia directly monitor the functional state of synapses in vivo and determine the fate of ischemic terminals. J Neurosci 29:3974-3980.

Wang ET, Sandberg R, Luo S, Khrebtukova I, Zhang L, Mayr C, Kingsmore SF, Schroth GP, Burge CB. 2008. Alternative isoform regulation in human tissue transcriptomes. Nature 456:470-476.

Wolf S, Barton D, Kottschade L, Grothey A, Loprinzi C. 2008. Chemotherapy-induced peripheral neuropathy: prevention and treatment strategies. Eur J Cancer 44:1507-1515. 